\title{
MODELING ONE AND TWO PASSIVE SCALAR MIXING IN TURBULENT JETS USING ONE-DIMENSIONAL TURBULENCE
}

\author{
MARTEN KLEIN ${ }^{1 *}$, CHRISTIAN ZENKER ${ }^{1}$, KATJA HERTHA ${ }^{1}$ AND \\ HEIKO SCHMIDT ${ }^{1}$ \\ ${ }^{1}$ Numerical Fluid and Gas Dynamics, Brandenburg University of Technology Cottbus-Senftenberg \\ Siemens-Halske-Ring 15A, D-03046 Cottbus, Germany \\ *Contact: marten.klein@b-tu.de • https://www.b-tu.de/en/fg-stroemungsmodellierung/
}

Key words: One-dimensional turbulence, passive scalar mixing, stochastic modeling, turbulent jet

\begin{abstract}
Turbulent mixing of two passive scalars is investigated in a constant-property jets using stochastic one-dimensional turbulence (ODT). Scalars are separately injected by a central round and a surrounding annular jet that issue into a uniform co-flow of low velocity. These scalars are transported downstream and dispersed in radial direction by turbulent advection and molecular diffusion. The jet as well as the turbulent inflow are numerically simulated with ODT as stand-alone tool using a temporal (T-ODT) and spatial (S-ODT) formulation. We show that ODT captures key properties of the turbulent mixing for one scalar by performing individual scalar statistics and for two scalars by computation of joint probabilities. Some limitations of the one-dimensional modeling approach are also discussed.
\end{abstract}

\section{INTRODUCTION}

Turbulent mixing is ubiquitous in flows from technical to astrophysical scales where it manifests itself by redistribution of conserved scalars on a range of scales. Reversible advective stirring motions of the turbulence cascade dominate down to the dissipation scales at which irreversible molecular mixing processes will take over. The transition from inertial to diffusive dominance is given by the Kolmogorov [1], Batchelor [2], and Obhukov-Corrsin [3] scales for the momentum, a slowly, and a fast diffusing scalar, respectively. An accurate representation of the flow physics down to these scales is particularly relevant whenever detailed information about the mean and fluctuations are required. This is, in particular, the case for turbulent flows with active scalars such as turbulent thermal or moist convection, as well as chemically reacting flows. For the latter, it is well known that the location and maximum of the heat release in a non-premixed jet flame, for example, crucially dependent on the mixing of the chemical species in the jet (e.g. $[4,5])$ which may be idealized to the mixing of one (e.g. [6, 7]) or multiple (e.g. $[8,9,10])$ passive scalars. Hence, any numerical approach that is deemed to have predictive capabilities needs to capture the mixing of passive scalars to facilitate or inhibit chemical reactions further downstream.

Direct numerical simulation (DNS) would be the ideal tool to numerically investigate the flows mentioned above since no modeling is involved but it is usually too costly to be broadly applicable. Reynoldsaveraged Navier-Stokes simulation (RANS) is economical but fluctuations have been filtered and only some effects are statistically modeled which strongly limits the predictive capabilities. Large-eddy simulation (LES) aims to capture a large fraction of relevant turbulent fluctuations. However, once small- 
scale processes are crucial for the application resolution requirements for LES tend toward those of DNS. Hence, we seek accurate but economical modeling approaches that allow for self-contained flow simulations by respecting fundamental physical principles.

The considerations above are addressed by lower-order stochastic modeling such as one-dimensional turbulence (ODT) [11]. ODT aims to represent all relevant scales of a turbulent flow for a one-dimensional computational domain. Deterministic molecular diffusion is directly resolved along this domain, whereas effects of turbulent advection are modeled by an ensemble of discrete mapping events (eddy events). This ensemble of eddy events covers a range of scales and is obtained by sampling individual eddy events from a stochastic process that depends on the evolving flow state. Notice that the latter distinguishes ODT from the linear eddy model (LEM) [12], in which scalars are mixed by a prescribed turbulence field (see also [13] for one and [10,14] for multiple scalar mixing in jets). For single passive scalar mixing it was previously shown that ODT is able to capture relevant scalar statistics for various canonical flows (e.g. $[15,16,17])$. By contrast, for three scalar mixing in isotropic turbulence some limitations related to the purely one-dimensional representation have been observed that manifest themselves by degraded joint probability density functions of the scalar state space [16]. The goal of this study therefore is to further investigate multiple scalar mixing but for nonhomogeneous, nonisotropic turbulence, which is somewhat closer to applications like jet flames (e.g. [4, 5]). Here we specifically consider two scalar mixing in a round jet as canonical case by utilizing ODT as stand-alone tool.

The rest of this paper is organized as follows. In section 2 we describe the model formulation for flows with mean cylindrical geometry. In section 3 we describe the selected round jet configuration and the

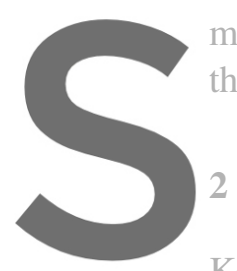
model application. In sdction 4 we present and disc
the round jets. At last, in section 5 we close with so
2 OVERVIEW OF THE CYIINDRICAL ODT

Kerstein's [11] one-dimensional turbulence (ODT) model aims to resolve all relevant scales of a turbulent flow which is made feasible by a dimensional model reduction. The effects of turbulent stirring

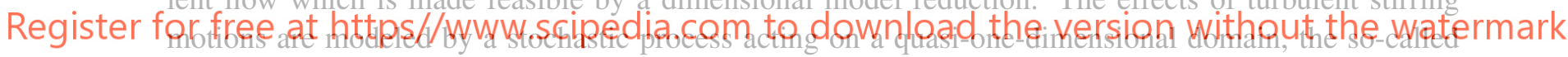

ODT domain, which is shown in figure 1 for the present application. Deterministic molecular diffusion is, hence, directily resolved along this domain.

The purpose of the cylindrical ODT formulation [18] is self-contained lower-order modeling of turbulent flows with axial symmetry of the mean like round jets and pipe flows. This model formulation is based on an adaptive Lagrangian finite-volume discretization [19] and is technically documented in [20]. We added passive scalars to the latter code as described in [17]. Below, some aspects of the model formulation are discussed, which are relevant for the model application to the mixing of passive scalars in round jets. We note that a complete model description is beyond the scope of this paper so that we defer the reader to [18] (and references therein) for details.

\subsection{Governing equations: T-ODT and S-ODT}

We distinguish two types of lower-order stochastic ODT equations describing momentum and scalar conservation in constant-property flows. In a temporal (T-ODT) model formulation, the ODT domain 
aims to resolve the temporal evolution of flow properties along the resolved direction. The lower-order equations are a set of 1-D partial differential equations (PDEs),

$$
\begin{aligned}
\frac{\partial \vec{u}}{\partial t}+\mathcal{E}_{\vec{u}} & =\frac{1}{r} \frac{\partial}{\partial r}\left(v r \frac{\partial \vec{u}}{\partial r}\right)-\vec{e}_{x} \frac{1}{\rho} \frac{\mathrm{d} P}{\mathrm{~d} x}, \\
\frac{\partial Y_{i}}{\partial t}+\mathcal{E}_{Y_{i}} & =\frac{1}{r} \frac{\partial}{\partial r}\left(\Gamma_{i} r \frac{\partial Y_{i}}{\partial r}\right),
\end{aligned}
$$

where $t$ denotes the time, $\vec{u}=\left(u_{x}, u_{r}, u_{\theta}\right)^{\mathrm{T}}$ the velocity vector with its conventional cylindrical components in axial $(x)$, radial $(r)$, and azimuthal $(\theta)$ direction, respectively, $\rho$ and $v$ the fluid's constant mass density and kinematic viscosity, respectively $\vec{e}_{x}(\mathrm{~d} P / \mathrm{d} x)$ a streamwise mean pressure gradient acting as a momentum source that is nonzero only for the generation of turbulent inflow, $Y_{i}$ is the mass fraction, and $\Gamma_{i}$ the molecular diffusivity of the $i$ th $(i=1,2, \ldots)$ conserved scalar due to division by $\rho$. In the equations above, $\mathcal{E}_{\vec{u}}$ and $\mathcal{E}_{Y_{i}}$ represents the effects of turbulent eddies for the velocity vector and a conserved scalar. Both terms are coupled together and formulated with the aid of discrete stochastic mappings, denoted as 'eddy events' that are described below. We only note here that $\mathcal{E}_{\vec{u}}$ and $\mathcal{E}_{Y_{i}}$ vanish between two subsequent eddy events which yields piecewise continuous advancement of parabolic PDEs representing deterministic molecular processes [11].

For T-ODT simulations of confined flows (like pipe flow), the ODT domain is located at a fixed position whereas, for jets, the domain is advected downstream as a whole. This is achieved by a global time-tospace transformation [4]
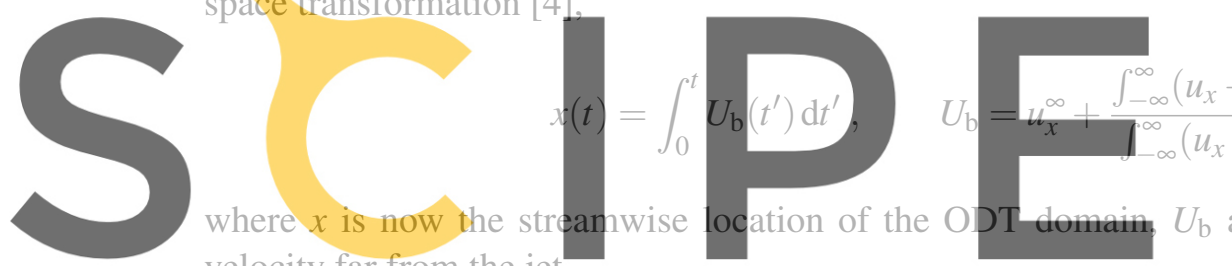
velocity far from the jet

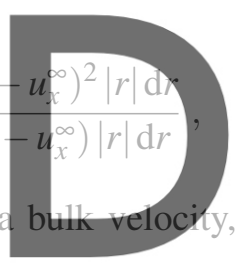
and $u_{x}$

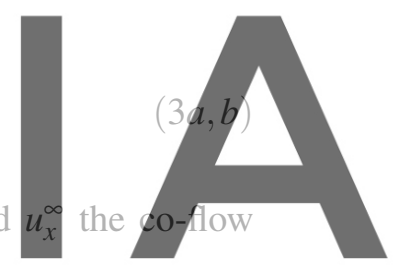

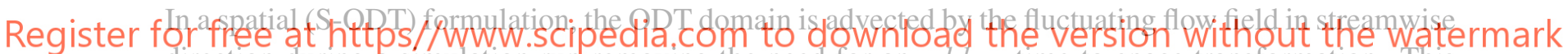
direction during a simulation run removing the need for an ad hoc time-to-space transformation. This formulation assumes a statistically stationary 2-D turbulent flow that will have a parabolic character in the model solution due to which it is determined by initial conditions, here to be taken as inflow. For S-ODT, the temporal rates of change of $\vec{u}$ and $Y_{i}$ in equations (1) and (2) are formally replaced by streamwise and radial advection terms. The mean radial advection occurs in jets as secondary flow due to streamwise mass flux conservation and momentum diffusion. Solutions of the S-ODT equations are parabolic yielding evolution along the streamwise coordinate $x$ instead of time $t$ during a simulation run,

$$
\begin{aligned}
& \frac{\partial\left(u_{x} \vec{u}\right)}{\partial x}+\left.\frac{1}{r} \frac{\partial\left(r u_{r} \vec{u}\right)}{\partial r}\right|_{\mathrm{LCF}}+\mathcal{E}_{\vec{u}}=\frac{1}{r} \frac{\partial}{\partial r}\left(r v \frac{\partial \vec{u}}{\partial r}\right)-\vec{e}_{x} \frac{1}{\rho} \frac{\mathrm{d} P}{\mathrm{~d} x}, \\
& \frac{\partial\left(u_{x} Y_{i}\right)}{\partial x}+\left.\frac{1}{r} \frac{\partial\left(r u_{r} Y_{i}\right)}{\partial r}\right|_{\mathrm{LCF}}+\mathcal{E}_{Y_{i}}=\frac{1}{r} \frac{\partial}{\partial r}\left(r \Gamma_{i} \frac{\partial Y_{i}}{\partial r}\right),
\end{aligned}
$$

where the subscript LCF ('Lagrangian cell faces') reminds us of the Lagrangian treatment of the radial advection term. The associated radial velocity $u_{r}$ is resolved by displacement of cell faces in the semidiscrete finite volume discretization of the governing equations (see figure 1 for a sketch of the quasi-1-D 
finite volume grid). Therefore, the Lagrangian treatment effectively decouples the PDEs by an exploit of the parabolic nature of the lower-order equations.

Note that the mean pressure gradient term in the above S-ODT equations is only needed for generation of turbulent inflow. Note further that the conserved scalars $Y_{i}$ are prescribed by the initial condition and have no internal sources or sinks in both T-ODT and S-ODT.

\subsection{ODT eddy events}

This section provides an overview of some relevant aspects of the ODT eddy event formulation but does not aim to be complete. For technical details we defer the reader to the literature cited.

In equations (1)-(5), $\mathcal{E}_{\vec{u}}$ represents the effects of turbulent advection and pressure fluctuations, whereas $\mathcal{E}_{Y_{i}}$ represents only the effects of turbulent advection. In order to distinguish velocity from scalars in the model, a mechanism for inter-component kinetic energy conversion has been introduced by [21]. This mechanism maximizes the inter-component kinetic energy exchange due to pressure-velocity couplings by a model parameter $\alpha$ that affects $\mathcal{E}_{\vec{u}}$ but not $\mathcal{E}_{Y_{i}}$. For $\alpha=0$ there is no redistribution of energy among the velocity components, for $\alpha=1$ energy redistribution is maximized, and for $\alpha=2 / 3$ equipartition is achieved, which maximizes the rate at which a return to small-scale isotropy is observed.

In the following we specialize to T-ODT. ODT eddy events are implemented as a stochastic sequence of mapping events that instantaneously manipulate property profiles of $\vec{u}(r)$ and $Y_{i}(r)$ by application of the triplet map $f(r)$ and kernel functions $K(r)=r-f(r)$ and $J(r)=|K(r)|$ for the map denoted triplet map B (TMB) in [18]. fluid from location $f(y)$ but introduces artificial di event is characterized by $t$, are sampled from an unknown probability density fu
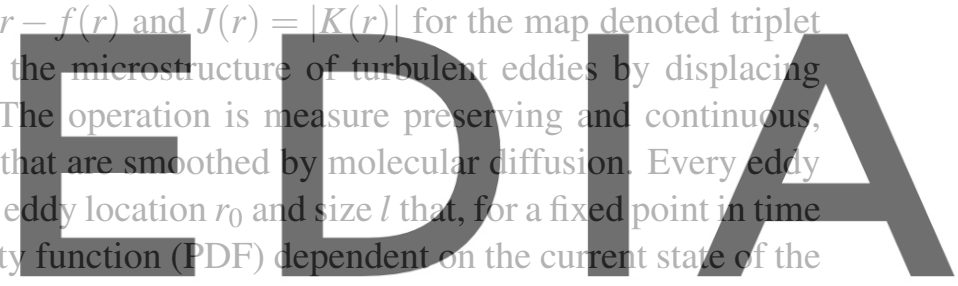

flow [11]. A costly construction of this PDF is avoided in practice by using a more efficient thinning-and-

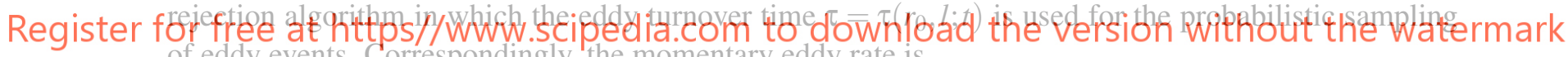
of eddy events. Correspondingly, the momentary eddy rate is

$$
\tau^{-1}=C \sqrt{\frac{2}{\rho V l^{2}}\left(E_{\mathrm{kin}}-Z E_{\mathrm{vp}}\right)},
$$

where $V$ is the notional eddy volume that is associated with $l$ at location $r_{0}, \rho$ the mass density, $E_{\text {kin }} \sim$ $\sum_{j=1}^{3} u_{K, j}^{2} / 2$ the shear-extractable kinetic energy due to mapping-kernel $K(r)$ weighted velocity components $u_{K, j}=\int u_{j} K r \mathrm{~d} r$, and $E_{\mathrm{vp}} \sim v^{2} / l^{2}$ a viscous penalty energy for the selected scale $l$. Some constant dimensional prefactors were dropped here but these are given in [18]. The coefficients $C$ and $Z$ in equation (6) denote the ODT turbulence intensity and the viscous suppression parameter, respectively. $C$ controls the mean rate of eddy events, whereas $Z$ provides an energy criterion for the smallest permissible eddy size. Physically plausible eddy events have real-valued $\tau$.

Note that in the S-ODT context, energy terms in equation (6) are reinterpreted as energy fluxes, while the eddy turnover time $\tau$ is superseded by a turnover length scale in streamwise direction. Either in T-ODT or S-ODT, equation (6) is evaluated for stochastic rejection (acceptance) of any candidate eddy event that has been economically obtained from guessed individual distribution functions for $l$ and $r_{0}$. 


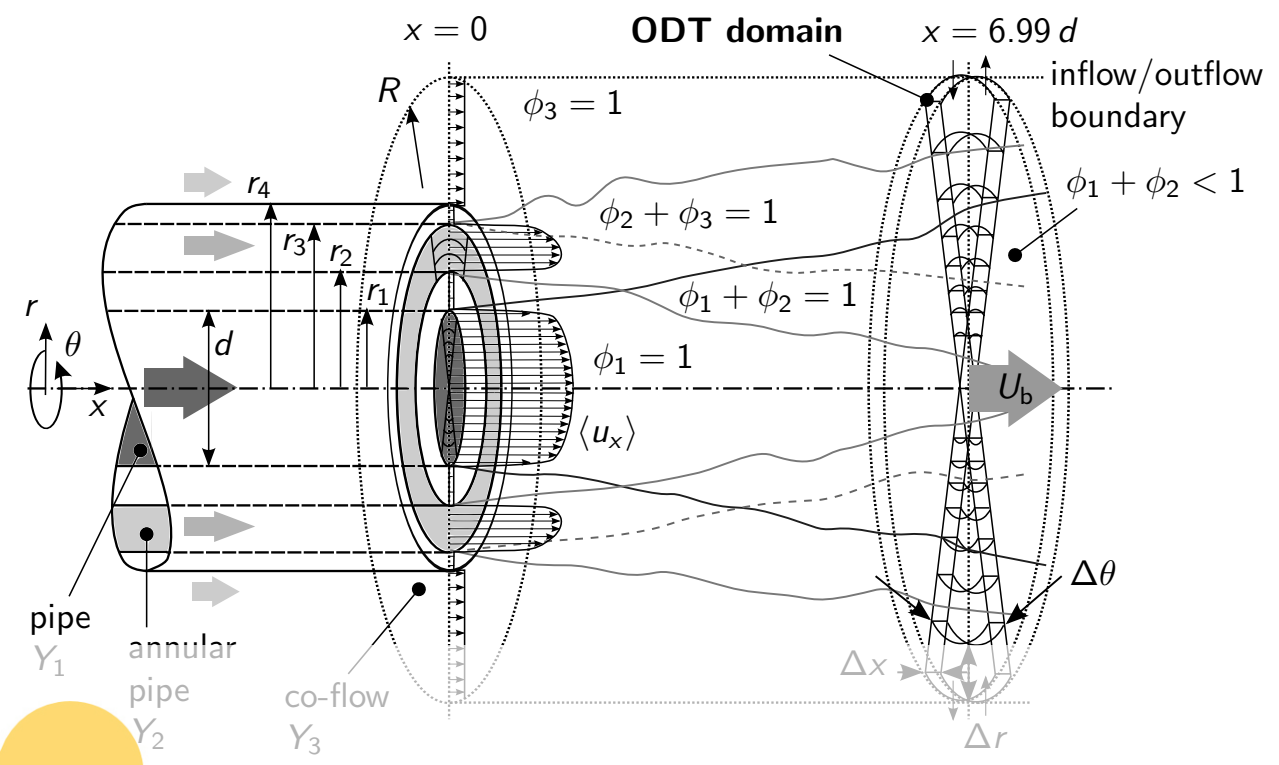

Figure 1: Sketch of the configuration investigated. Turbulent round and coaxial jets issue into a slow co-flow at location $x=0$ yielding the mean velocity profile $\left\langle u_{x}\right\rangle$. Each of the three inflow streams contains a passive scalar with initially uniform mass fraction $Y_{i}$ or mixture fraction $\phi_{i}(i=1,2,3)$. Numerical simulations of the jet evolution are performed by ODT for which a quasi-one-dimensional domain spans the diameter and is advected downstream (in $x$ direction) with bulk velocity $U_{b}$ during a simulation run that solves parabolized conservation equations. The ODT domain is divided into Lagrangian finite volumes $\Delta V=r \Delta r \Delta \theta \Delta x$ ) of finite radiaz $(r)$ but infinitesimal ax al
$(x)$ and azimuthal $(\theta)$ size. The turbulent inflow condition at $x=0$ is generated from stano-alone pipe and annular
pipe flow simulations, respectively, whereas the co-flow is uniform.
3 MODEL APPLICATION TO THE ROUND JET

In this section we describe the case set-up, the application of the ODT model to the jet, and the generation

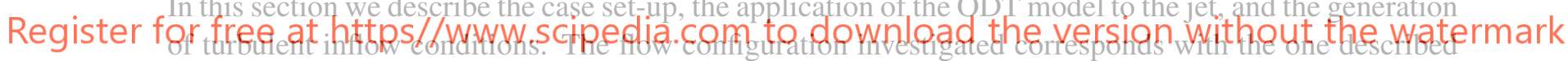
in $[8,9]$.

\subsection{A three-stream round jet configuration}

Figure 1 shows the flow configuration investigated together with some details of the inflow condition and the ODT domain orientation. A central round and a coaxial annular jet of low Mach number issue into a slow co-flow at atmospheric standard conditions at axial location $x / d=0$, where $d=5.54 \mathrm{~mm}$ is the inner diameter of central incoming pipe that, in the following, serves as reference length scale. The incoming jets are turbulent and obtained with T-ODT simulations as discussed below in section 3.2. We therefore concentrate on the round-jet set-up for the rest of this section.

Following [8], the central jet has bulk velocity $U_{1}=34.5 \mathrm{~m} / \mathrm{s}$ and consists of an acetone-air mixture with acetone mass fraction $Y_{1}<1$ but scalar mixture fraction $\phi_{1}=1$, mass density $\rho_{1}=1.26 \mathrm{~kg} / \mathrm{m}^{3}$, kinematic viscosity $v_{1}=1.340 \times 10^{-5} \mathrm{~m}^{2} / \mathrm{s}$, and scalar diffusivity $\Gamma_{1}=1.039 \times 10^{-5} \mathrm{~m}^{2} / \mathrm{s}$ (acetone in air) yielding the Schmidt number $S c_{1}=v_{1} / \Gamma_{1}=1.29$. The annular jet has bulk velocity $U_{2}=32.5 \mathrm{~m} / \mathrm{s}$ and consists of 
ethylene with mass fraction $Y_{2}=1$ and mixture fraction $\phi_{2}=1$, mass density $\rho_{2}=1.14 \mathrm{~kg} / \mathrm{m}^{3}$, kinematic viscosity $v_{2}=0.830 \times 10^{-5} \mathrm{~m}^{2} / \mathrm{s}$, and scalar diffusivity $\Gamma_{2}=1.469 \times 10^{-5} \mathrm{~m}^{2} / \mathrm{s}$ (ethylene in air) yielding $S c_{2}=v_{2} / \Gamma_{2}=0.565$. The co-flow has bulk velocity $U_{3}=0.4 \mathrm{~m} / \mathrm{s}$ and consists of ambient air with mass fraction $Y_{3}=1$ and mixture fraction $\phi_{3}=1$, mass density $\rho_{3}=1.17 \mathrm{~kg} / \mathrm{m}^{3}$, and kinematic viscosity $v_{3}=1.59 \times 10^{-5} \mathrm{~m}^{2} / \mathrm{s}$. Note that co-flow air is the 'background fluid' and not an additional scalar so that entrainment from the co-flow dilutes the two scalars $Y_{1}$ and $Y_{2}$. Note further that the small co-flow velocity assures existence of the parabolic flow solution.

The incoming jets are separated by walls shown in figure 1 . We account for the finite thickness of the walls separating the three inflow streams. For the radially oriented ODT domain, the geometry is fully defined by the pipe inner radius $r_{1}=d / 2=2.770 \mathrm{~mm}$, the annulus inner radius $r_{2}=3.175 \mathrm{~mm}$, the annulus outer radius $r_{3}=4.190 \mathrm{~mm}$, the co-flow inner radius $r_{4}=4.75 \mathrm{~mm}$, and the outer radius $R \gg r_{4}$ of the domain. In the reference experiments [8], the co-flow is bounded by an external pipe with radius $R=75 \mathrm{~mm}$ that we also use for the ODT domain. This domain is radially open in contrast to the experiments in order to facilitate radial mass, momentum, and energy exchange with the co-flow within the parabolic ODT equations.

Stochastic ODT simulations of the turbulent jet are carried out for the quasi-one-dimensional ODT domain as sketched in figure 1 by dropping the mean pressure gradient term $\mathrm{d} P / \mathrm{d} x=0$ in equations (1) and (4). This domain moves downstream with the flow as described above in section 2. It remains aligned with the radius at all times in T-ODT as well as S-ODT and spans the whole diameter, that is, $r \in[-R, R]$. Negative $r$ are necessary in order to avoid an artificial transport barrier at the axis that would bias the

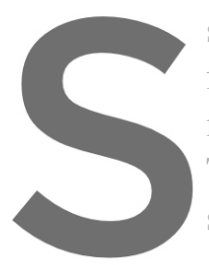
solution [18]. Assuming universality of the turbule
from [18] that were obtained by calibration of the vel
measurements yielding $C=5.25, Z=400$, and $\beta_{\mathrm{LS}}$
T-ODT based on a planar jet [17]. The difference $\mathrm{i}$
spatial sampling. We select $\alpha=0$ for simplicity and
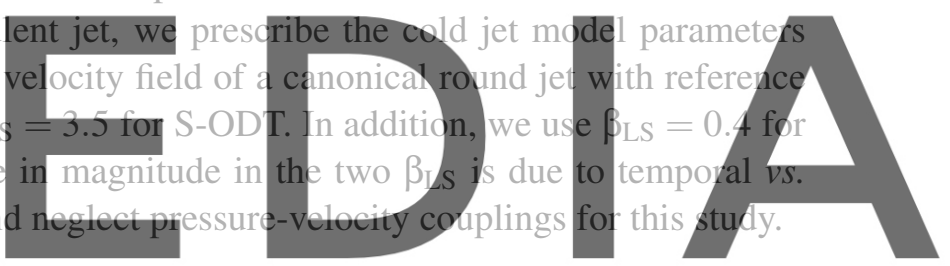

Register for. 3 ODT as turbullent inflow generator

Register for free at https//WwW.scipedia.com to download the version without the watermark

The 'natural' approach for the preparation of turbulent inflow conditions is a self-contained one. We use

T-ODT as infiow generator and apply it separately to the turbulent pipe and annular pipe fiows in order to generate $N$ ensembles of uncorrelated flow profiles in order to join them with the co-flow in order to prescribe the inflow as initial flow profile in the ODT round jet simulations. For pipe flow, the ODT domain crosses the pipe diameter similar to the jet, whereas it covers only a positive radial interval for the annular pipe as sketched in figure 1. No-slip, zero-flux boundary conditions are prescribed for the walls located at $r_{k}(k=1,2,3,4)$. A constant mean pressure gradient is prescribed to drive the flow. In the statistically stationary state, this forcing is eventually balanced on average by frictional losses.

In order to generate turbulent inflow conditions from pipe flow, we set the ODT model parameters to $C=5, Z=350, \alpha=0$ (instead of $2 / 3$ to be consistent with the jet), and large-eddy suppressions are switched off by selecting $\beta_{\mathrm{LS}}=0$ [18]. The largest permissible eddy size is $l_{\max } / d=1 / 3$, which is less than the value of $1 / 2$ used for channel flow [22] as a consequence of 'geometric lensing' that influences large and near-axis eddy events. For annular pipe flow, the starting point is closer to moderate $R e$ number channel flow so that we select $C=10$ and $Z=600$ [19] but account for the radial asymmetry because 

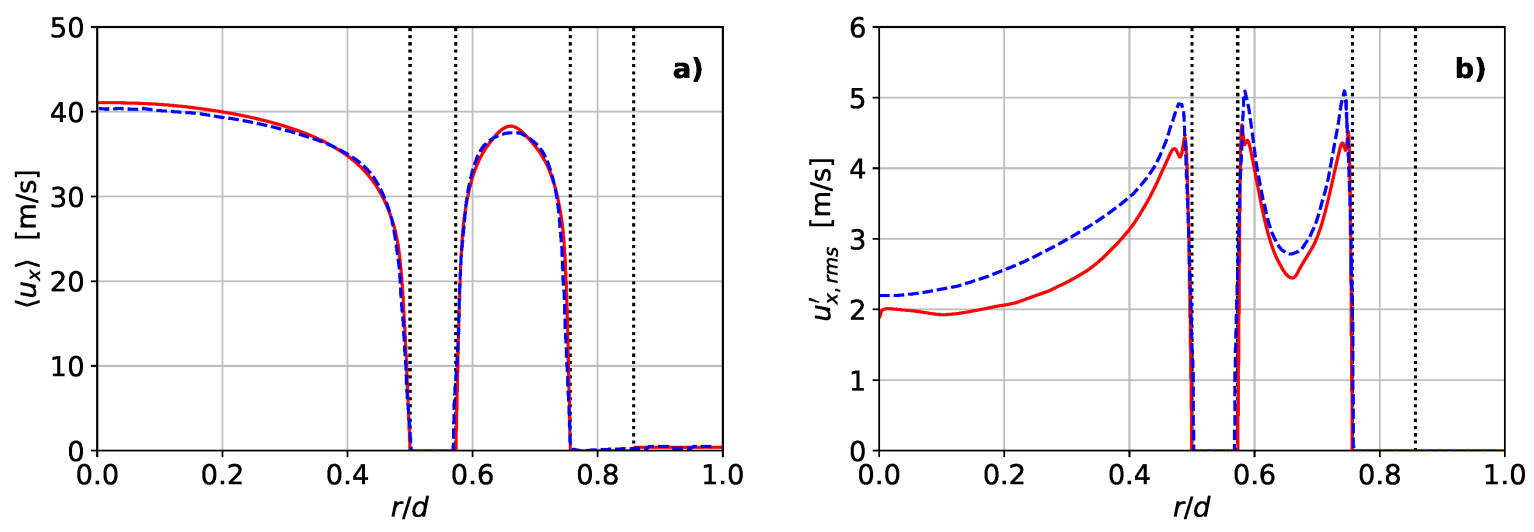

ODT ---- RANS

Figure 2: Statistics of the inflow at $x / d=0$ showing the mean (a) and root-mean-square (b) streamwise velocity that are consistent with reference RANS results from [9].

of the radius ratio $\eta=r_{2} / r_{3} \approx 3 / 4<1$. Since $l_{\max } / L=1 / 2$ with $L=r_{3}-r_{2}$ for $\eta=\rightarrow 1$ (channel) and $l_{\max } / L=1 / 3$ for $\eta \rightarrow 0$ (pipe), we select $l_{\max } / L=2 / 3$.

Figure 2 shows radial profiles of the mean $\left\langle u_{x}\right\rangle$ and root-mean-square (r.m.s.) $u_{x, \text { rms }}^{\prime}=\sqrt{\left\langle u_{x}^{2}\right\rangle-\left\langle u_{x}\right\rangle^{2}}$

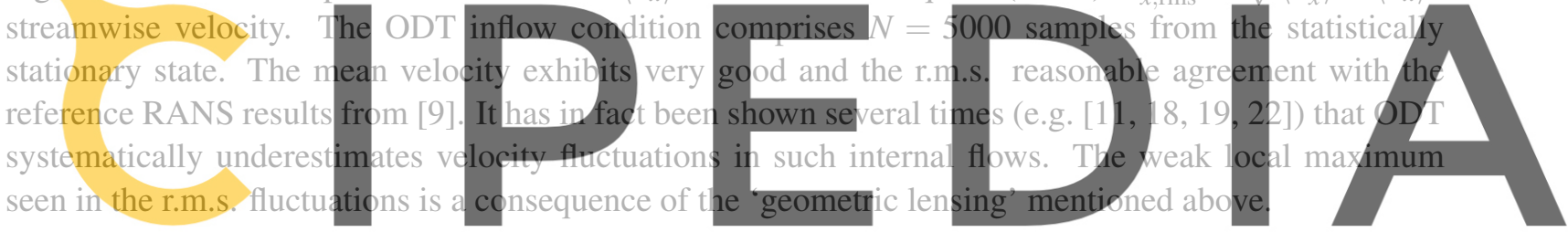

\section{Register for frESUtThttps//www.scipedia.com to download the version without the watermark}

In this section we show ODT results for two passive scalars in the round jet from figure 1. We begin with conventional statistics and then proceed with the joint probability density functions (jPDFs).

\subsection{Axial profiles of the scalar mean and fluctuation variance}

The following analysis is based on locally normalized scalar concentrations utilizing the dimensionless mixture fraction $\phi_{i}$ of the $i$ th scalar that is defined as

$$
\phi_{i}=Y_{i} / \sum_{j=1}^{3} Y_{j} \quad \text { for } \quad i=1,2,3,
$$

where $Y_{i}=c_{i} / \rho$ is the dimensionless mass fraction due to a scalar concentration $c_{i}\left[\mathrm{~kg} / \mathrm{m}^{3}\right]$. Here we are interested in the mixing of acetone $(i=1)$ and ethylene $(i=2)$ in air $(i=3)$ so that we consider only $\phi_{1}$ and $\phi_{2}$ for the analysis. It is worth noting that the sum of the mixture fractions is conserved, i.e., $\phi_{1}+\phi_{2}+\phi_{3}=1$, which implies $\phi_{i} \in[0,1] \forall i$ since only two of the scalars are independent. 

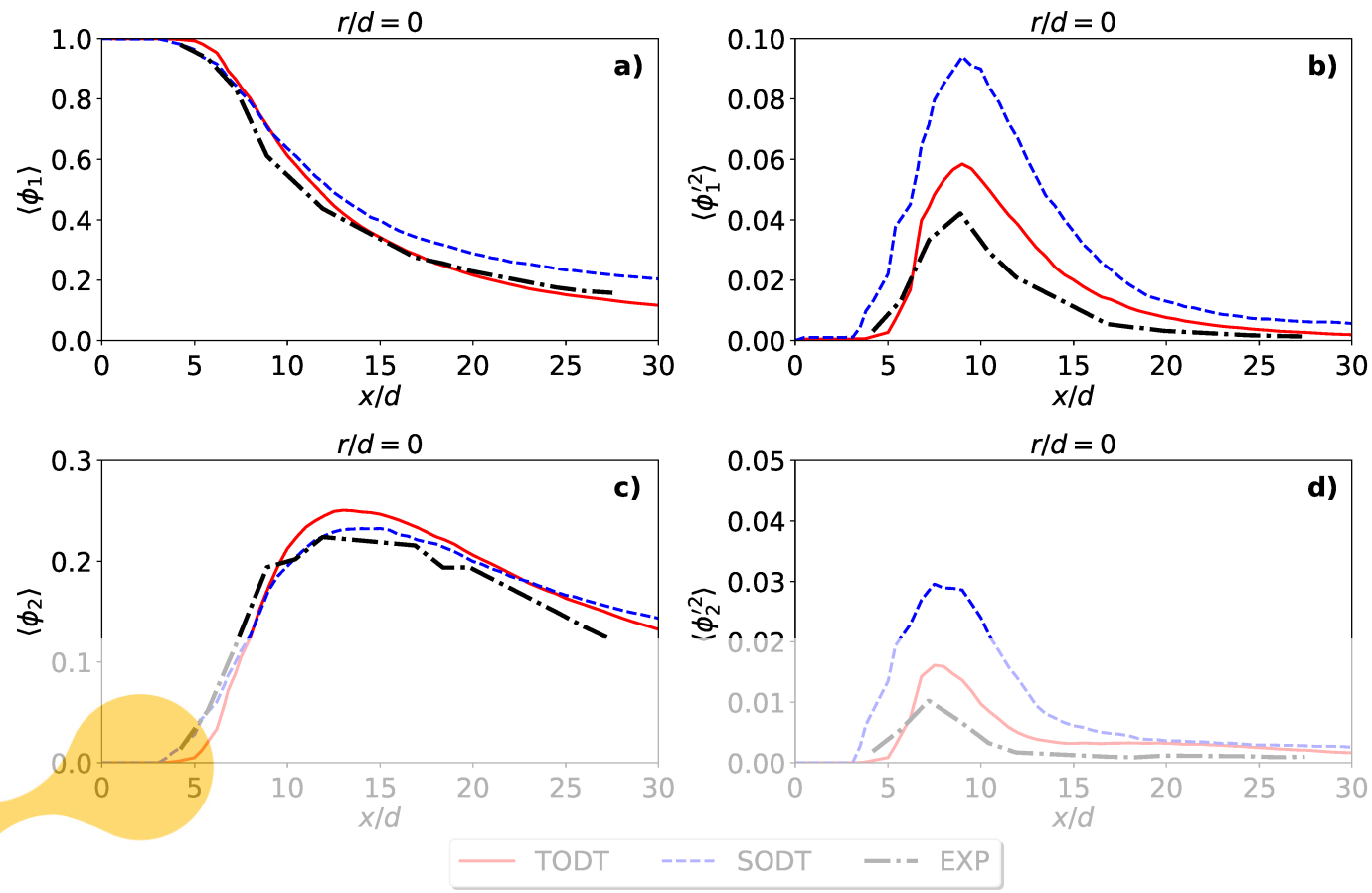

Figure 3: Centerline (axial $x$ ) profiles of the mean $(\mathrm{a}, \mathrm{c})$ and fluctuation variance $(\mathrm{b}, \mathrm{d})$ of the normalized passive scalar concentrations $\phi_{1}$

S-ODT are shown togethe

Conventional ensemble av scalar mixture fraction
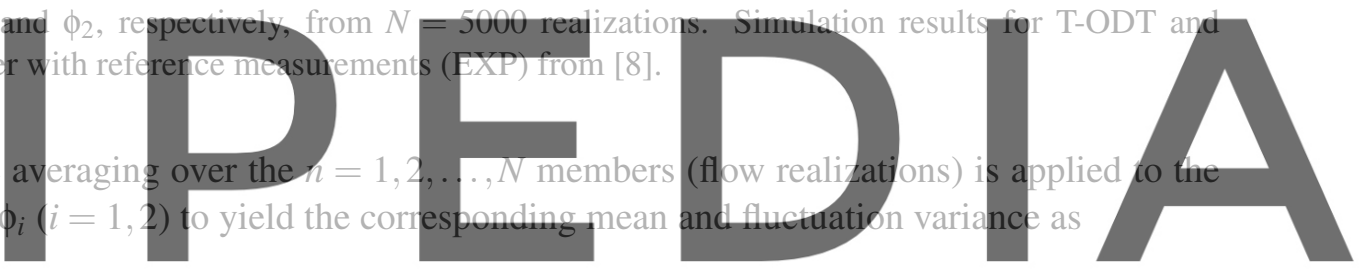

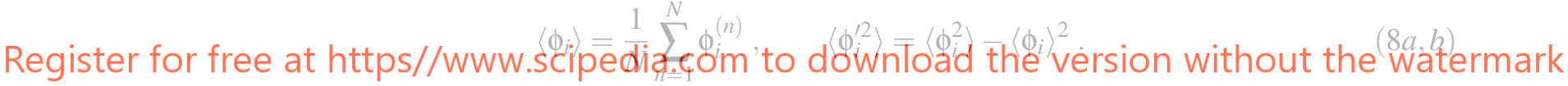

Figure 3 shows axial profiles of the scalar mean $\left\langle\phi_{i}\right\rangle(x / d)$ and fiuctuation variance $\left\langle\phi_{i}^{\prime 2}\right\rangle(x / d)$ for the centerline $r / d=0$. T-ODT and S-ODT simulation results from $N=5000$ realizations are shown together with reference measurements (EXP) from [8]. The mean field shown in figure 3(a,c) exhibits good agreement between the reference, T-ODT, and S-ODT results. For both T-ODT and S-ODT the onset of mixing $(x / d \approx 5)$ is delayed. While $\left\langle\phi_{1}\right\rangle$ decays a bit too slowly in S-ODT, T-ODT overestimates the maximum of $\left\langle\phi_{2}\right\rangle$. The level of agreement nevertheless shows that the modeling approach is generally robust. It has good predictive capabilities since no model parameter was changed since the calibration for a canonical round jet with bulk Reynolds number $\operatorname{Re}=U d /(2 v) \approx 47,000$ [18]. In the present application, we do not only face the additional annular jet but also much smaller Reynolds numbers, that is, $R e_{1}=U_{1} r_{1} / v_{1}=7130$ for the central round and $R e_{2}=U_{2}\left(r_{3}-r_{2}\right) / v_{2}=3974$ for the annular jet.

The results discussed so far indicate only a weak sensitivity of the mean field to details of the turbulence dynamics. We therefore turn to the fluctuations in order to investigate this further. Figure 3(b,d) shows the fluctuation variance $\left\langle\phi_{i}^{\prime 2}\right\rangle$ for $i=1,2$. Quantitative agreement between the reference, T-ODT, and SODT results can only be discerned for the location of the fluctuation maxima that line up roughly between 

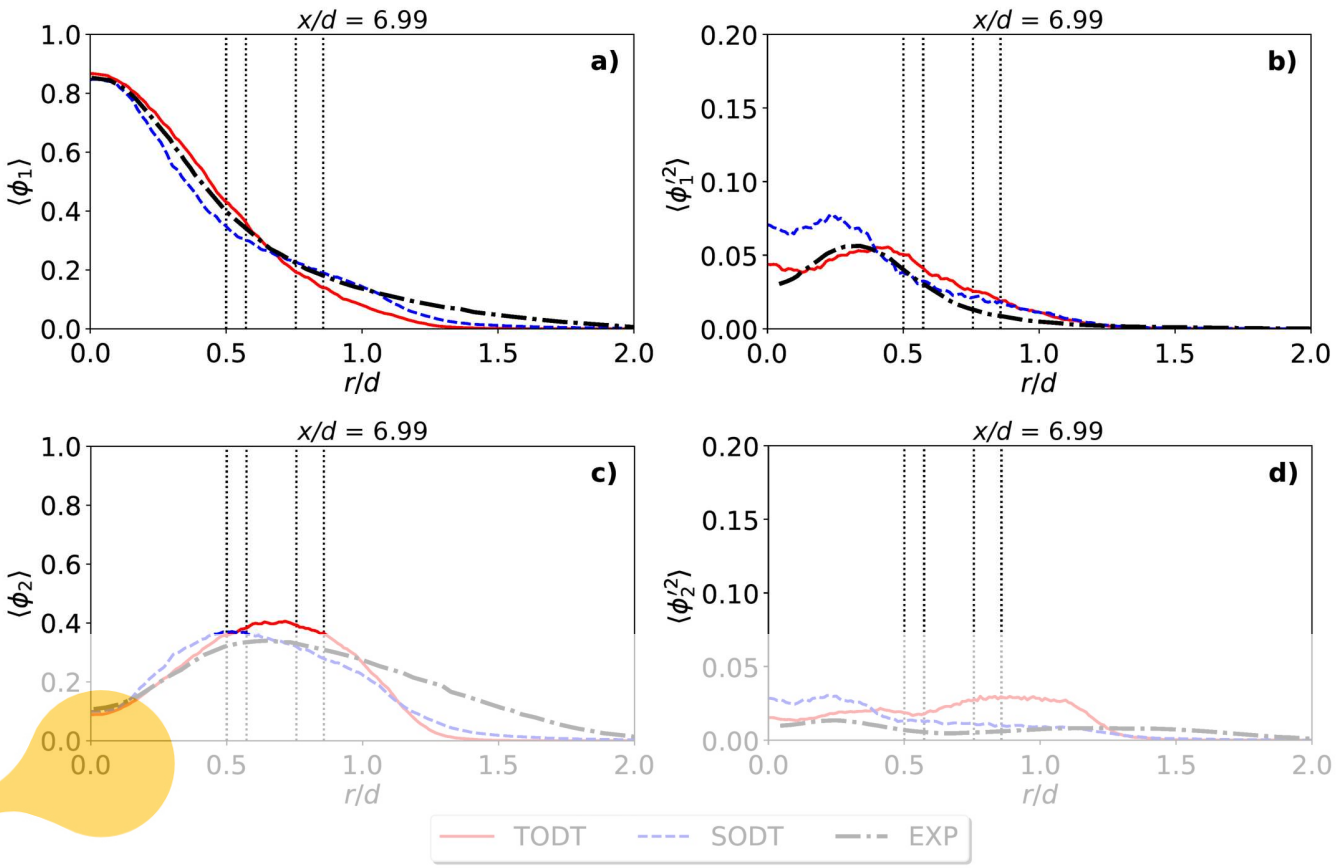

Figure 4: Radial $(r)$ profiles of the mean $(a, c)$ and fluctuation variance $(b, d)$ of the normalized passive scalar concentrations $\phi_{1}$ and $\phi_{2}$, respectively, from $N=5000$ realizations at downstream location $x / d=6.99$. Simulation results for T-ODT and S-ODT are shown together with reference measurements (EXR) from [8]. Dotted verical lines at $r_{k} / d(k=1,2,3,4)$ correspond to the stream edges in the infow plane $(\boldsymbol{d}=0)$ a orientation.

$x / d \approx 9$ and 7 for $i=1$ and $i=2$, respectively. Otherwise, only quali

the various results. The local fluctuation maximum is overestimated by a factor of $\approx 1.5$ in T-ODT

by $\approx 2.5$ in S-ODT. This is primarily. due to 'geometric lensing' effects near the axis as described in

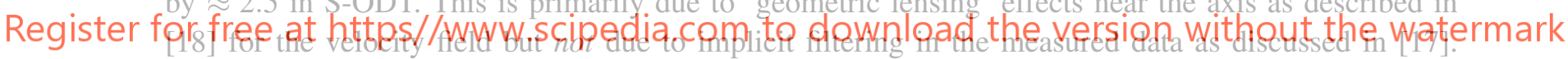

Here, Schmidt numbers are $O(1)$ so that Kolmogorov scale resolution in reference measurements [8] is sufficient to accurately resolve the scaiar fluctuations.

\subsection{Radial profiles of the scalar mean and fluctuation variance}

Figure 4 shows radial profiles of the scalar mean $\left\langle\phi_{i}\right\rangle(r / d)$ and fluctuation variance $\left\langle\phi_{i}^{\prime 2}\right\rangle(r / d)$ for downstream location $x / d=6.99$ in the vicinity of the axial scalar fluctuation maximum. The mean field is shown in figure $4(\mathrm{a}, \mathrm{c})$ and exhibits good to reasonable agreement between reference, T-ODT, and S-ODT results. For these radial profiles, both T-ODT and S-ODT results notably diverge from the reference results of [8] for $r / d \gtrsim 1$, but S-ODT is able to capture the large $r$ tails a bit better than T-ODT due to relatively longer acting molecular diffusion in regions with low velocity.

Next, radial profiles of the fluctuation variance are shown in figure $4(b, d)$ in which S-ODT results are in good agreement with reference data for $r / d \gtrsim 0.3$, whereas T-ODT results overshoot for $\left\langle\phi_{2}^{\prime 2}\right\rangle$. Closer to the axis we can discern an artificial increase of the scalar fluctuations. This is consistent with the artificial 

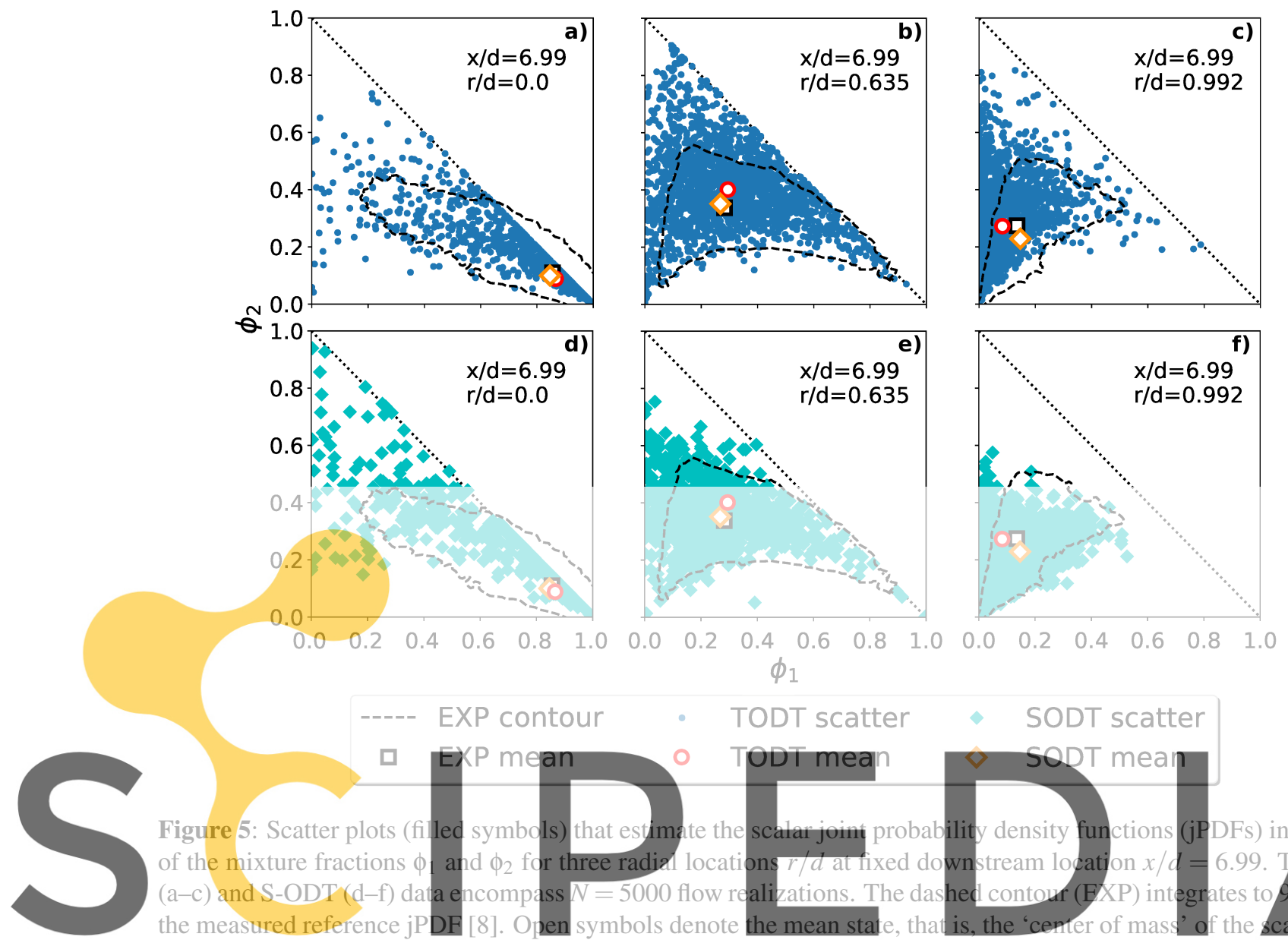

$\square \quad$ EXP mea

Figure 5: Scatter plots (filled symbols) that estimate the

of the mixture fractions $\phi$

$(\mathrm{a}-\mathrm{c})$ and S-ODT $(\mathrm{d}-\mathrm{f})$ data encompass

the measured reference jPDF
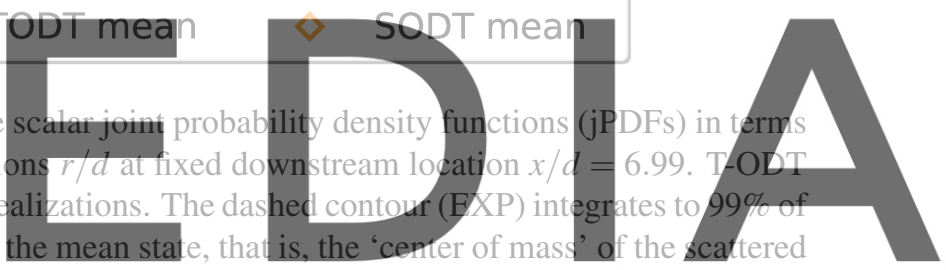

data points. The triangular region above the diagonal is inaccessible for passive scalars in constant-property flow.

Register for free at https//www.scipedia.com to download the version without the watermark increase of the velocity variance in the vicinity of the axis as discussed in [18] for the mappings denoted TMA, TMB (used here), and PTMB in light of the coordinate singularity at $r / d=0$.

\subsection{Scalar joint probability density functions}

Figure 5 shows T-ODT (upper row) and S-ODT (lower row) scatter plots of instantaneous mixture fractions $\phi_{1} v s . \phi_{2}$ for an ensemble of $N=5000$ flow realizations. The number of data points contained in some state-space volume serves may serve as estimator for the joint probability density which would require larger $N$. The scatter plots obtained with ODT are shown in comparison to the isoline of the 99\% percentile of the measured reference jPDF [8] at downstream location $x / d=6.99$ for three radial positions $r / d=0,0.635,0.992$. Additionally, the mean ('center of mass') of each ODT scatter plot and corresponding reference jPDF are given by open symbols. The area above the diagonal is not accessible.

Going from the left to the right panel in each row of figure 5 we 'move' radially outward. For $r / d=0$ (at the axis) the jPDF is localized in the vicinity of the unmixed center jet state $\left(\phi_{1}=1, \phi_{2}=0\right)$. For $r / d=0.635$ (roughly in the middle of the incoming annular jet) the jPDF is widely spread but on average 
only weak entrainment is notable due to $\left\langle\phi_{1}\right\rangle \approx\left\langle\phi_{2}\right\rangle \approx 0.35$ yielding $\left\langle\phi_{1}\right\rangle+\left\langle\phi_{2}\right\rangle=O(1)$. For $r / d=0.992$ (outside of the incoming annular jet) the scalars are diluted but have notably dispersed into the co-flow air since $\left\langle\phi_{1}\right\rangle \approx\left\langle\phi_{2}\right\rangle \approx 0.2$ yielding $\left\langle\phi_{1}\right\rangle+\left\langle\phi_{2}\right\rangle\langle O(1)$ Furthermore, good agreement is obtained between the shapes of the measured jPDFs and the S-ODT simulation results shown. By contrast, present T-ODT results seem to generally exhibit a larger variability than S-ODT and the reference data but the model fidelity is only weakly reduced. Near the axis $(r / d \ll 1)$ both T-ODT and S-ODT exhibit much larger variability albeit the scatter plots visually overemphasize rare events. The mean values, however, are only weakly affected by the fluctuation details.

\section{CONCLUDING REMARKS}

Stochastic numerical simulations of the turbulent mixing of two passive scalars in a turbulent round jet have been performed using an adaptive cylindrical formulation of one-dimensional turbulence (ODT). In general, ODT aims to resolve all relevant scales of a turbulent flow within a dimensionally reduced setting. Molecular diffusion is directly resolved, whereas the effects of turbulent advection are modeled by a stochastic process. For the coaxial round jet investigated, we have shown that the strength of ODT lies in the economical and accurate modeling of the state-space of scalar fluctuations despite the fact that the mean scalar profiles had some shortcomings. Fluctuation capture, however, is a more relevant model property for model application to multiphysics turbulent flows. The fidelity of the temporal (TODT) and spatial (S-ODT) model formulations was demonstrated for a moderate Reynolds and Schmidt number regime by profiles of the scalar fluctuation variance and joint probability density functions. These diagnostic quantities tend to be better captured by S-ODT than T-ODT by taking into account not only a local diffusive but also a local advective scale velocity. Limitations of the quasi-1-D modeling approach manifest themselves mainly in the vicinity of the axis which is a consequence of the polar coordinates (see [18] for elaboration for the velocity that analogously affects conserved scalars). Altogether, the degree of the turbulent fluctuation capture provided by ODT is beyond the scope of RANS and would normally require high-resolution LES or DNS. This suggests that ODT may be useful for reaching into highly turbulent flow regimes that are inaccessible to the latter two methods.

\section{REFERENCES}

[1] Kolmogorov, A.N. The local structure of turbulence in incompressible viscous fluid for very large Reynolds numbers. Dok. Akademii Nauk SSSR (1941) 30:299-303.

[2] Batchelor, G.K. Small-scale variation of convected quantities like temperature in turbulent fluid. Part 1. General discussion and the case of small conductivity. J. Fluid Mech. (1959) 5:113-133.

[3] Corrsin, S. On the spectrum of isotropic temperature fluctuations in isotropic turbulence. J. Appl. Phys. (1951) 22:469-473.

[4] Echekki, T., Kerstein, A.R., Dreeben, T.D., and Chen, J.-Y. 'One-dimensional turbulence' simulation of turbulent jet diffusion flames: Model formulation and illustrative applications. Combust. Flame (2001) 125:1083-1105.

[5] Cabra, R., Chen, J.-Y., Dibble, R.W., Karpetis, A.N., and Barlow, R.S. Lifted methane-air jet flames in a vitiated coflow. Combust. Flame (2005) 143:491-506. 
[6] Eswaran, V., and Pope, S.B. Direct numerical simulations of the turbulent mixing of a passive scalar. Phys. Fluids (1988) 31:506-520.

[7] Dowling, D.R., and Dimotakis P.E. Similarity of the concentration field of gas-phase turbulent jets. J. Fluid Mech. (1990) 218:109-141.

[8] Cai, J., Dinger, M.J., Li, W., Carter, C.D., Ryan, M.D., and Tong, C. Experimental study of threescalar mixing in a turbulent coaxial jet. J. Fluid Mech. (2011) 685:495-531.

[9] Rowinski, D.H., and Pope, S.B. An investigation of mixing in a three-stream turbulent jet. Phys. Fluids (2013) 25:105105.

[10] Sannan, S., Weydahl, T., and Kerstein, A.R. Stochastic simulation of scalar mixing capturing unsteadiness and small-scale structure based on mean-flow properties. Flow Turbulence Combust. (2013) 90:189-216.

[11] Kerstein, A.R. One-dimensional turbulence: model formulation and application to homogeneous turbulence, shear flows, and buoyant stratified flows. J. Fluid Mech. (1999) 392:277-334.

[12] Kerstein, A.R. A linear-eddy model of turbulent scalar transport and mixing. Combust. Sci. Technol. (1988) 60:391-421.

[13] Kerstein, A.R. Linear-eddy modeling of turbulent transport. Part 3. Mixing and differential molecular diffusion in round jets. J. Fluid Mech. (1990) 216:411-435.

[14] Kerstein, A.R. Linear-eddy modelling of turbulent transport. Part 7. Finite-rate chemistry and multistream mixing. J. Fluid Mech. (1992) 240:289-313.

[15] Ashurst, W.T., Kerstein, A.R., Pickett, L.M., and Ghandhi, J.B. Passive scalar mixing in a spatially developing shear layer: Comparison of one-dimensional turbulence simulations with experimental results. Phys. Fluids (2003) 15:579-582.

[16] Giddey, V., Meyer, D.W., and Jenny, P. Modeling three-dimensional scalar mixing with forced one-dimensional turbulence. Phys. Fluids (2018) 30:125103.

[17] Klein, M., Zenker, C., and Schmidt, H. Small-scale resolving simulations of the turbulent mixing in confined jets using one-dimensional turbulence. Chem. Eng. Sci. (2019) 204:186-202.

[18] Lignell, D.O., Lansinger, V.B., Medina, J., Klein, M., Kerstein, A.R., Schmidt, H., Fistler, M. and Oevermann, M. One-dimensional turbulence modeling for cylindrical and spherical flows: model formulation and application. Theor. Comput. Fluid Dyn. (2018) 32:495-520.

[19] Lignell, D.O., Kerstein, A.R., Sun, G. and Monson, E.I. Mesh adaption for efficient multiscale implementation of one-dimensional turbulence. Theor. Comput. Fluid Dyn. (2013) 27:273-295.

[20] Stephens, V.B. and Lignell, D.O. Computationally efficient modeling and simulation of turbulent flows. SoftwareX (2021) 13:100641.

[21] Kerstein, A.R., Ashurst, W.T., Wunsch, S., and Nilsen, V. One-dimensional turbulence: vector formulation and application to free shear flows. J. Fluid Mech. (2001) 447:85-109.

[22] Schmidt, R.C., Kerstein, A.R., Wunsch, S., and Nilsen, V. Near-wall LES closure based on onedimensional turbulence modeling. J. Comput. Phys. (2003) 186:317-355. 